\title{
130 SECOND RING-DOWN TIME AND 3.98 MILLION QUALITY FACTOR IN 10 KHZ FUSED SILICA MICRO BIRDBATH SHELL RESONATOR
}

Tal Nagourney, Jae Yoong Cho, Ali Darvishian, Behrouz Shiari, and Khalil Najafi ${ }^{*}$

Center for Wireless Integrated MicroSensing and Systems (WIMS ${ }^{2}$ )

University of Michigan, Ann Arbor, MI, USA

\begin{abstract}
We achieved a 130.0 second ring-down time constant for the $9.736 \mathrm{kHz} n=2$ wine glass mode of a birdbath shell microresonator, corresponding to a quality factor of 3.98 million. The resonator is a fused silica shell with an outer radius of $2.5 \mathrm{~mm}$, anchor radius of $0.5 \mathrm{~mm}$, height of $\sim 2.1 \mathrm{~mm}$, and thickness ranging from $\sim 15-70 \mu \mathrm{m}$. This is the longest reported ring-down time and quality factor to date for any micro resonator at this frequency. We also measured an extremely low $138 \mathrm{mHz}$ frequency split between wine glass modes at $10.389 \mathrm{kHz}$ with a low damping mismatch of $7.1 \times 10^{-5} \mathrm{~Hz}$. This unprecedented result is a considerable achievement for microresonators and will pave the way to the development of next generation navigation-grade MEMS gyroscopes with $10 \mathrm{e}-3^{\circ} / \mathrm{hr}$ bias stability.
\end{abstract}

\section{INTRODUCTION}

Great advancement in the performance of MEMS gyroscopes has been achieved over the past two decades, yet they are still not suitable for dead-reckoning of drones, self-driving cars, and pedestrians, which require very high bias stability $\left(<0.1^{\circ} / \mathrm{h}\right)$. The vibratory decay time constant $(\tau)$ is an important parameter that directly affects bias stability (bias stability $\propto \tau^{-1}[1]$ ). Unfortunately, $\tau$ of silicon gyroscopes is typically limited to $<5 \mathrm{~s}$ due to large thermoelastic damping (TED) at frequencies greater than $10 \mathrm{kHz}$. A quad mass silicon gyroscope with $\tau=181 \mathrm{~s}(Q=$ 1.21 million) at $2.1 \mathrm{kHz}$ has been reported [2]; however, it has potentially high vibration sensitivity since its frequency is close to that of external noise sources.

Over the past few years, our group has demonstrated a fused silica micro birdbath (BB) resonator gyroscope [3], [4]. The BB resonator is a significant step toward achieving a low-cost microscale navigation-grade MEMS gyroscope. Compared to other existing micro shell resonators such as [5] or [6], the BB resonator has several key advantages for achieving both very long $\tau$ and small $\Delta f$.

First, the anchor and the rest of the resonator are self-aligned. That is, the shell is defined from a single reflow-molding step using a radially symmetric mold. Misalignment between the anchor and rim is known to be a key source of anchor loss; the BB resonator achieves low anchor loss by eliminating this misalignment.

Second, the $\mathrm{BB}$ resonator has a long, smooth transition region that connects the rim to the anchor. This long transition region isolates the part with the most mechanical energy (rim) and the energy sink (anchor). The smooth curvature reduces the concentration of mechanical stress near the anchor. Both of these features effectively trap the mechanical energy in the rim, greatly reducing energy leakage to the anchor.

Third, the micro blowtorching process can form BB resonators out of fused silica (FS) - which has one of the lowest amounts of thermoelastic damping of any material-and results in an ultra smooth surface quality. Other researchers have demonstrated a structure similar to the birdbath resonator that achieved quality factor $(Q)$ of 1.05 million [7], but due to its high $105 \mathrm{kHz}$ frequency it only produced $\tau$ of $3.18 \mathrm{~s}$. A slightly longer 4.32 s $\tau$ was reported in [6] for a cylindrical microcrystalline diamond shell resonator, with a frequency of $23.07 \mathrm{kHz}$ and $Q$ of 313,100 . A higher frequency $160 \mathrm{kHz}$ hemispherical resonator gyroscope with a $9.0 \mathrm{~s} \tau(Q=4.5$ million) was reported in [8].

We initially reported the micro fused silica (FS) birdbath (BB) resonator (Figure 1) and accompanying micro-blowtorch molding process (Figure 3) to form BB shells with thermal reflowmolding at $\sim 1600{ }^{\circ} \mathrm{C}$ [9]. In [10] we first reported demonstration of the $\mathrm{BB}$ resonator as a gyroscope. The resonator operates in the $n=$ 2 wine glass (WG) modes; in rate-mode, the gyroscope is operated in force-to-rebalance mode, while in rate-integrating mode the vibrations are allowed to freely precess around the rim, their orientation indicating the rotated angle. We have designed the BB resonator geometry to place the desired $n=2$ wine glass modes around $10 \mathrm{kHz}$ to avoid environmental noise, and as far as possible from parasitic modes to prevent interference. More detail on the $\mathrm{BB}$ resonator gyroscope can be found in [4].

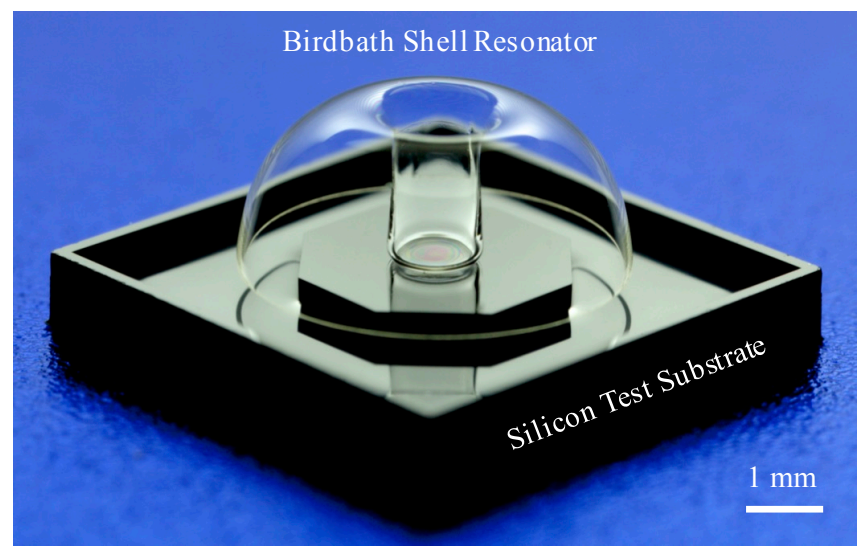

Figure 1: Fused silica birdbath resonator resting on a silicon base. We have measured $\tau$ of $130.0 \mathrm{~s}$ and $Q$ of 3.98 million at $9.736 \mathrm{kHz}$ for an $n=2$ wine glass mode of this type of resonator.

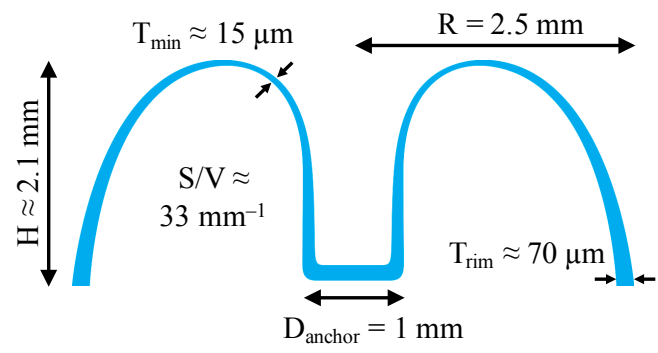

Figure 2: Cross sectional profile of a birdbath resonator showing relevant dimensions and approximate thickness variation due to reflow molding, as well as its high surface-to-volume $(\mathrm{S} / \mathrm{V})$ ratio.

Recently, we made significant progress in perfecting the fabrication process and achieved a record $\tau$ of $130.0 \mathrm{~s}$ and $Q$ of 3.98 million at $9.736 \mathrm{kHz}$, a huge improvement from $8.3 \mathrm{~s}$ at $\sim 8.2$ $\mathrm{kHz}$ [9]. Based on the analysis on bias stability and $\tau$ in [1], we estimate our reported $\tau$ will enable an ultra-high bias stability $<10 \mathrm{e}-3^{\circ} / \mathrm{h}$ in future gyroscopes. In this paper we describe several 
key improvements in the $\mathrm{BB}$ resonator fabrication process that allowed us to achieve this level of performance.

\section{FABRICATION}

An overview of the fabrication process is provided here, but for a detailed discussion on the principles of fused silica birdbath resonator fabrication, refer to [11].

\section{Molding the Shell}

Individual birdbath shells are molded by thermal reflow from $\sim 100$ - $\mu \mathrm{m}$-thick $1.5 \times 1.5 \mathrm{~cm}^{2}$ fused silica substrates. The substrates are placed atop a graphite mold with a 2.5 -mm-radius cylinder machined out from its middle and a 0.5 -mm-radius post left standing in the center of the cylinder. To rapidly reach the high softening temperature of fused silica, $\sim 1600{ }^{\circ} \mathrm{C}$, a propane-oxygen blowtorch is lowered from above by a motorized Z-stage. With minimal contact - only at the anchor post and outer wall-the softened fused silica substrates reflow into the desired shape. The final $\mathrm{BB}$ resonator shape is therefore not explicitly defined by the mold; the outer mold wall determines the major radius and the central post defines the anchor, but the curvature and overall shape are determined by pressure and temperature during the $<10 \mathrm{~s}$ molding process. This process is depicted in Figure 3. We have performed extensive finite element modeling to improve our understanding of the impact of each process condition on the final resonator shape [12], [13].

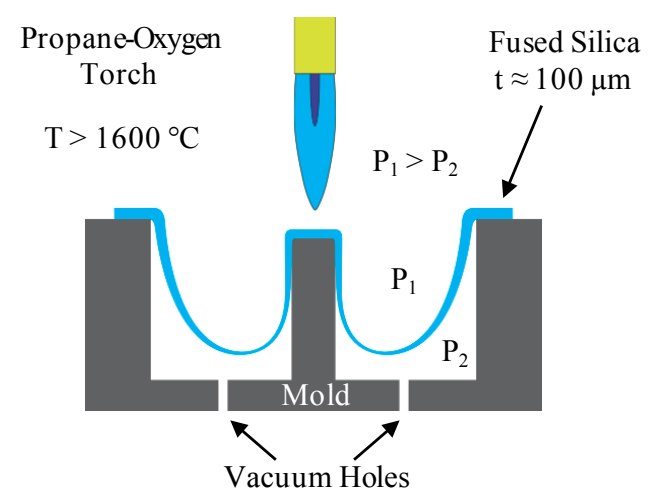

Figure 3: Thermal reflow of a 100- $\mu$ m-thick fused silica substrate into a graphite mold to form a birdbath resonator. The pressure inside the mold cavity $\left(P_{2}\right)$ is lower than the outside pressure $\left(P_{1}\right)$ [11]. The reflow process takes $<10 \mathrm{~s}$.

\section{Shell Separation and Polishing}

The reflowed substrate now consists of a square frame with a molded birdbath shell protruding from its center. The square frame must be removed to isolate the shell resonator. This is accomplished by lapping and chemical mechanical polishing (CMP). A thick silicon lapping jig wafer with an array of through holes protects the shells while their frames are removed. The shells sit below the surface of the jig wafer and are encapsulated in thermoplastic polymer for protection. The square frame sits on the surface, leveling the shells to the wafer. As the wafer is lapped, the frame is removed. A CMP step completes the fabrication, polishing the rims of the shells. This process is depicted in Figure 4. The shells are then released and cleaned in a solvent bath. A photograph of a completed resonator is shown in Figure 1 and relevant dimensions are given in Figure 2.

\section{Metallization}

If the birdbath shell is to be used as a gyroscope, a conductive film is applied to its surface to enable electrostatic drive and readout of its rim motion. This is typically accomplished by sputtering a gold film over a chromium or titanium adhesion layer.

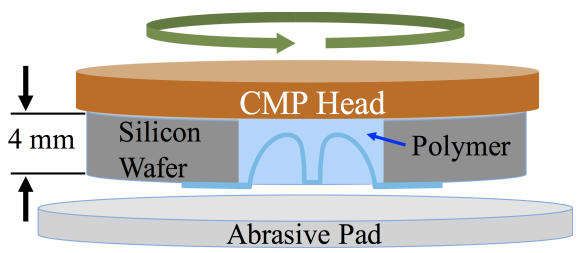

Figure 4: After molding, birdbath shells are protected with thermoplastic polymer within a jig wafer so their square frames can be removed by lapping and their rims polished with CMP.

\section{TESTING}

To prepare a birdbath resonator for testing, its anchor is attached with glass frit to a raised pedestal on a silicon substrate. The silicon substrate is then temporarily attached to a piezoelectric actuator with a thermoplastic polymer and placed into a vacuum chamber that is evacuated to $<10 \mu$ Torr (Figure 5). The actuator drives the resonator with sinusoidal vibrations at its resonant frequency along its axis of radial symmetry; the vibrations couple into the desired $n=2$ wine glass modes. Each of the two modes, in turn, are excited into resonance, then the drive signal is cut and the resonator is allowed to ring down. The motion of the rim is measured with a Polytec laser Doppler velocimeter (LDV) through a window in the vacuum chamber, as depicted in Figure 5. This technique works well for metallized or uncoated fused silica resonators.

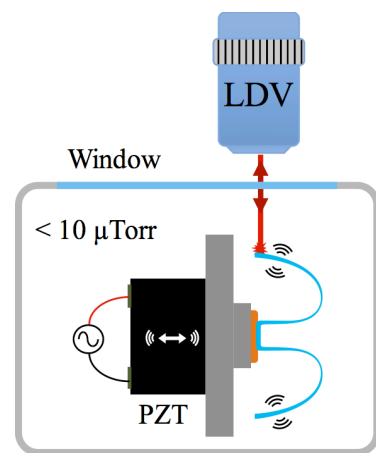

Figure 5: A PZT transducer drives the shell into resonance before it freely rings down. A laser Doppler velocimeter measures motion of $B B$ shell rim through a window in the vacuum chamber.

\section{RESULTS AND DISCUSSION}

Our birdbath resonators, whose natural $n=2$ wine glass mode frequencies are around $10 \mathrm{kHz}$, routinely have quality factors over one million and frequency splits between the two modes in the low tens of Hz. This outstanding performance is unprecedented for shell-microresonators, and even more impressive considering their rapid and relatively simple fabrication process. For an uncoated $\mathrm{BB}$ resonator, we achieved an extremely long $\tau$ of $130.0 \mathrm{~s}$ at $f_{1 n=2}$ $=9.736 \mathrm{kHz}(Q=3.98$ million) for one mode (Figure 6) and 115.1 $\mathrm{s}$ at $f_{2 \_n=2}=9.776 \mathrm{kHz}(Q=3.54$ million $)$ for the second mode. Implemented as a gyroscope this resonator should achieve an outstanding bias stability of $<10 \mathrm{e}-3^{\circ} / \mathrm{h}$, according to Challoner et al.'s analysis [1]. This marks a considerable improvement over our previous works, which most recently reported a 43 second $\tau$ at 8.8 $\mathrm{kHz}$ for a birdbath resonator.

The frequency split between wine glass modes, $\Delta f$, is also 
important for gyroscope performance. While it is possible to cancel the frequency split by electrostatically tuning stiffness, it is preferable to address this during fabrication. We measured an extremely low as-fabricated $\Delta f$ of $138 \mathrm{mHz}$ for a $\mathrm{BB}$ resonator at $10.389 \mathrm{kHz}$. This device has relatively long ring-down times of $\tau_{l}$ $=76.4 \mathrm{~s}\left(Q_{1}=2.49\right.$ million $)$ and $\tau_{2}=76.0 \mathrm{~s}\left(Q_{2}=2.48\right.$ million $)$ and a low damping mismatch $\left(\Delta \tau^{-1}\right)$ of $7.1 \times 10^{-5} \mathrm{~Hz}$. This low $\Delta f$ and $\Delta \tau^{-1}$ indicate a high degree of radial symmetry that should minimize anchor loss. The fact that our longest $\tau$ comes from a device with a $40 \mathrm{~Hz} \Delta f$ indicates that anchor loss may not be the dominant mechanism of energy loss for the BB resonator. In fact, we have not seen a strong correlation between $\Delta f$ and $\tau$, leading us to conclude that $\mathrm{BB}$ resonators are somewhat tolerant to molding variations.

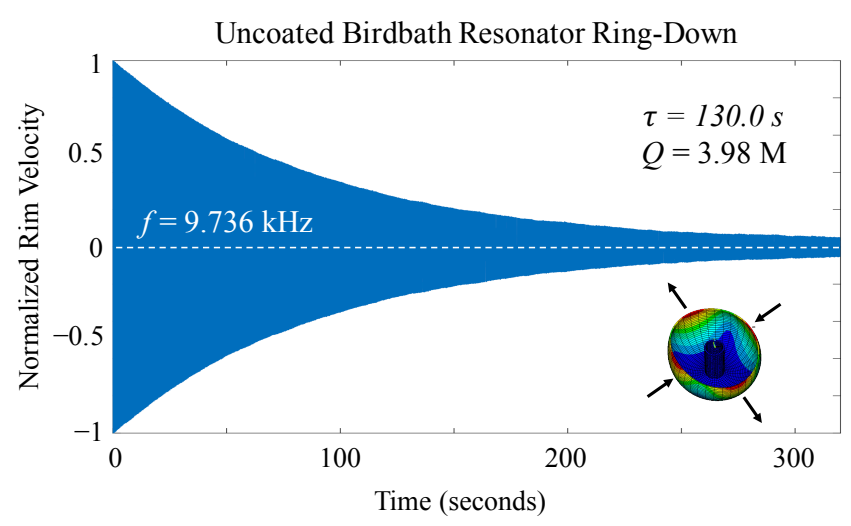

Figure 6: Ring-down plot of an uncoated birdbath resonator with our longest recorded $\tau=130.0 \mathrm{~s}$. The other mode at $9.776 \mathrm{kHz}$ has $\tau=115.1 \mathrm{~s}(Q=3.54$ million $)$. Results measured with $L D V$.

\section{Radial Symmetry}

We attribute our experimental results to improvements in our fabrication process. Maintaining a high degree of radial symmetry is essential for maximizing $Q_{\text {anchor }}$ of the $\mathrm{BB}$ resonator. It is designed so that the anchor point is a vibrational node, but any deviation from symmetry causes the nodal point to shift. In some cases we have seen a warped upper anchor region. This is the area that stretches the most and becomes the thinnest during molding, so it can be challenging to maintain a smooth transition from anchor to shell. This must be controlled, because if the radial symmetry is disrupted, the resonator will likely suffer increased anchor loss and damping mismatch between WG modes. Warping and imbalance also result in a frequency split between wine glass modes, which is problematic for gyroscope operation. The thermal distribution within the mold must be uniform, which requires a flame that will uniformly heat the mold as well as ensuring that the flame and mold are aligned.

\section{Rim Polishing}

After lapping away the square frame of fused silica from which a birdbath resonator is molded, the rim of the shell is quite rough. We have experimentally determined that roughness, scratches, and chips may limit performance and that the rim must be polished using CMP in order to reach a high $Q$. A high quality, defect-free rim is achieved by fine-tuning the pressures, rates, and times of the lapping and CMP processes, and by selection of an appropriate thermoplastic to protect the shells.

\section{Surface Quality}

We have observed experimentally that devices with more microscopic defects such as etch pits tend to have relatively low $Q$. There are several papers showing that the $Q \mathrm{~s}$ of fused silica resonators are inversely proportional to their surface-to-volume $(\mathrm{S} / \mathrm{V})$ ratios due to energy loss mechanisms pertaining to the surface [14]-[17]. The BB resonator may be especially sensitive to the condition of its surface considering its high $\mathrm{S} / \mathrm{V}$ ratio of $\sim 33$ $\mathrm{mm}^{-1}$. Fortunately, the micro blowtorch process smoothes out the surface during reflow to an average roughness of $1.8 \AA$ (Figure 7).

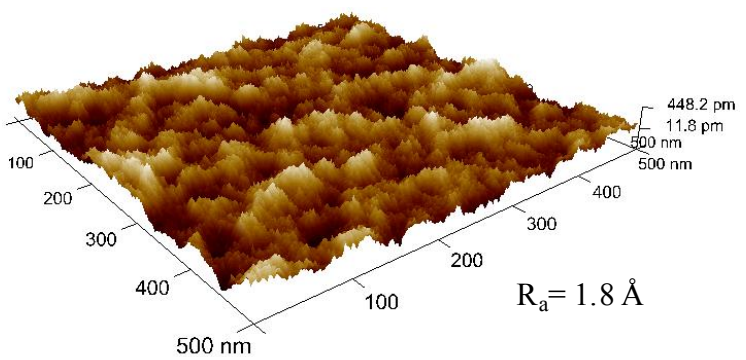

Figure 7: This AFM scan shows the smooth fused silica surface produced by thermal reflow molding with a blowtorch [18].

\section{Anchor Mounting}

The birdbath resonator is designed with an automatically centered anchor post that helps to minimize anchor loss. As mentioned earlier, the center of the anchor is a nodal vibration point, which helps reduce sensitivity to the anchor mounting condition. Still, a rigid anchor is required to minimize motion at the attachment point, which would lead to anchor loss. We have found that glass frit works well for this purpose.

\section{Conductive Coating}

Our $130.0 \mathrm{~s} \tau$ was measured for a bare resonator with LDV, but to implement a $\mathrm{BB}$ resonator as a gyroscope we must drive and read out its motion electrostatically. This requires a conductive surface, typically sputtered gold with a chromium adhesion layer. Figure 8 shows the effect of sputtering $\mathrm{Cr} / \mathrm{Au}$ on the top surface of each of two previously uncoated $\mathrm{BB}$ resonators. The initial deposition of $15 / 20 \AA \mathrm{Cr} / \mathrm{Au}$ considerably decreases $\tau$, but consecutive $40 \AA \mathrm{Au}$ depositions have a decreasingly strong effect. This suggests that the nature of the initial film is most important, rather than the overall thickness. A more complete discussion with additional details on this study can be found in [18].

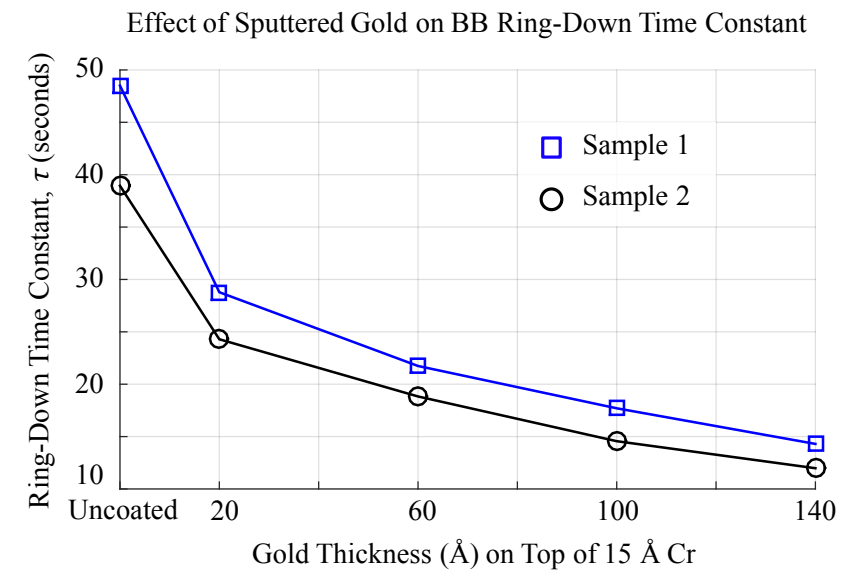

Figure 8: Coating 15/20 A Cr/Au on two previously uncoated $B B$ resonators considerably decreases their $\tau$. Adding additional $40 A$ layers of Au further decreases $\tau$, but less severely [18]. 
Based on this analysis, we are therefore developing techniques to maximize $\tau$ for metallized microresonators, including optimizing metal coverage and thickness and experimenting with other materials and deposition techniques. One attractive option is platinum atomic layer deposition (ALD). ALD enables conformal deposition of highly uniform thin films over corners and high aspect ratio features [19]. This should enable us to use a thinner film than would be required with sputtering to produce sufficient conductivity, thus maintaining the highest possible $\tau$ for a packaged gyroscope. We estimate from finite element modeling (FEM) that we are not limited by anchor loss or TED, and that resonators with optimized processing - even with a metal coating-will reach $Q \approx 6.8$ million $(\tau \approx 215 \mathrm{~s})$.

\section{CONCLUSION}

The results presented in this paper represent a major step forward in the development of high-performance MEMS gyroscopes. We have demonstrated the ability to precisely fabricate fused silica micro-shells and control their form to the extent necessary to produce a very high 3.98 million $Q$ and extremely long $130 \mathrm{~s} \tau$ near our target frequency of $10 \mathrm{kHz}$. We predict that a gyroscope with this performance will reach $10 \mathrm{e}-$ $3^{\circ} / \mathrm{hr}$ bias stability. We achieved these results by optimizing our fabrication process to improve radial symmetry, rim polishing, surface quality, and anchor attachment.

Although birdbath resonators are molded individually, the blowtorch molding process takes less than $10 \mathrm{~s}$ and we are able to rapidly produce devices. Resonant frequency and frequency split between wine glass modes are reproducible, especially for devices from the same mold. Furthermore, adjusting parameters such as shell depth can be easily performed during a fabrication run in order to fine-tune the shape and operational characteristics of the shells. This flexible yet controllable technique has enabled us to produce very high-performance resonators with fused silica that would not be possible with conventional micromachining.

\section{ACKNOWLEDGEMENTS}

This research is supported by the DARPA MRIG Program (\#W31P4Q-11-1-0002). Part of this work was performed in the Lurie Nanofabrication Facility (LNF); special thanks to all the staff, especially Pilar Herrera-Fierro and Tom Latowski for CMP support. Thank you to Scott Webster for machining graphite molds and to Robert Gordenker for supporting this research in the lab.

\section{REFERENCES}

[1] A. D. Challoner, H. H. Ge, and J. Y. Liu, "Boeing Disc Resonator Gyroscope," in IEEE/ION PLANS, Monterey, CA, 2014, pp. 504-514.

[2] S. Askari, M. H. Asadian, K. Kakavand, and A. M. Shkel, "Vacuum sealed and getter activated MEMS Quad Mass Gyroscope demonstrating better than 1.2 million quality factor," in IEEE Int. Symp. on Inertial Sensors and Systems, Laguna Beach, CA, 2016, pp. 142-143.

[3] K. Najafi and J. Cho, "Gyroscope and method of fabricating a resonator for a gyroscope," U.S. Patent 2013/0160578 A1, June 27, 2013.

[4] J. Cho, J. Woo, J. Yan, R. L. Peterson, and K. Najafi, "FusedSilica Micro Birdbath Resonator Gyroscope ( $\mu$-BRG)," $J$. $M E M S$, vol. 23, no. 1, pp. 66-77, Feb. 2014.

[5] P. Shao, L. D. Sorenson, X. Gao, and F. Ayazi, "WineglassOn-a-Chip," in Solid-State Sensors, Actuators and Microsystems Conf., Hilton Head, SC, 2012, pp. 275-278.

[6] H. Najar, C. Yang, A. Heidari, H. Najar, L. Lin, and D. A.
Horsley, "Batch-fabricated High Q-factor Microcrystalline Diamond Cylindrical Resonator," Proc. IEEE Int. Conf. $M E M S$, Estoril, 2015, pp. 801-804.

[7] D. Senkal, M. J. Ahamed, M. H. A. Ardakani, S. Askari, and A. M. Shkel, "Demonstration of 1 Million Q-Factor on Microglassblown Wineglass Resonators With Out-of-Plane Electrostatic Transduction," J. MEMS, vol. 24, no. 1, pp. 2937, Feb. 2015.

[8] A. Vafanejad and E. S. Kim, "Effect of diaphragm perforation on quality factor of hemispherical resonator gyroscope," in Transducers-18th Int. Conf. on Solid-State Sensors, Actuators and Microsystems, Anchorage, AK, 2015, pp. 27-30.

[9] J. Cho, J. Yan, J. A. Gregory, H. Eberhart, R. L. Peterson, and K. Najafi, "High-Q Fused Silica Birdbath and Hemispherical 3-D Resonators Made by Blow Torch Molding," in IEEE 26th Int. Conf. on MEMS, Taipei, 2013, pp. 177-180.

[10] J. Cho, J.-K. Woo, J. Yan, R. L. Peterson, and K. Najafi, “A high-Q birdbath resonator gyroscope (BRG)," in Transducers \& Eurosensors XXVII: The 17th Int. Conf. on Solid-State Sensors, Actuators and Microsystems, Barcelona, 2013, pp. 1847-1850.

[11] J. Cho, J. Yan, J. A. Gregory, H. W. Eberhart, R. L. Peterson, and K. Najafi, "3-Dimensional Blow Torch-Molding of Fused Silica Microstructures," J. MEMS, vol. 22, no. 6, pp. 12761284, Dec. 2013.

[12] B. Shiari, A. Darvishian, T. Nagourney, J. Cho, and K. Najafi, "A Comparison Between Experiments and FEM Predictions for Blowtorch Reflow of Fused Silica Micro-Shell Resonators," in Transducers-18th Int. Conf. on Solid-State Sensors, Actuators and Microsystems, Anchorage, AK, 2015, pp. 776-779.

[13] B. Shiari, T. Nagourney, A. Darvishian, J. Yoong Cho, and K. Najafi, "Numerical prediction of stress evolution during blowtorch reflow of fused silica micro-shell resonators," in IEEE Int. Symp. on Inertial Sensors and Systems, Laguna Beach, CA, 2016, pp. 13-16.

[14] A. M. Gretarsson and G. M. Harry, "Dissipation of mechanical energy in fused silica fibers," Rev. Sci. Instrum., vol. 70, no. 10, p. 4081-4087, 1999.

[15] S. D. Penn, G. M. Harry, A. M. Gretarsson, S. E. Kittelberger, P. R. Saulson, J. J. Schiller, J. R. Smith, and S. O. Swords, "High quality factor measured in fused silica," Rev. Sci. Instrum., vol. 72, no. 9, pp. 3670-3673, 2001.

[16] A. Ageev, B. C. Palmer, A. De Felice, S. D. Penn, and P. R. Saulson, "Very high quality factor measured in annealed fused silica," Class. Quantum Gravity, vol. 21, no. 16, pp. 3887-3892, Jul. 2004.

[17] S. D. Penn, A. Ageev, D. Busby, G. M. Harry, A. M. Gretarsson, K. Numata, and P. Willems, "Frequency and surface dependence of the mechanical loss in fused silica," Phys. Lett. A, vol. 352, no. 1-2, pp. 3-6, Mar. 2006.

[18] T. Nagourney, J. Cho, A. Darvishian, B. Shiari, and K. Najafi, "Effect of Metal Annealing on the Q-Factor of Metal-Coated Fused Silica Micro Shell Resonators," in IEEE Int. Symp. on Inertial Sensors and Systems Proceedings, Hapuna Beach, HI, 2015, pp. 1-5.

[19] R. L. Kubena, D. T. Chang, and R. L. Larson, "Quartz-based disk resonator gyro with ultra-thin conductive outer electrodes and method of making same," U.S. Patent 8766745 B1, July 1,2014 .

\section{CONTACT}

*K. Najafi, tel: +1-734-764-3317; najafi@umich.edu

T. Nagourney, tel+1-734-904-4666; taln@umich.edu 